\title{
Informática Educativa y Educación en Informática
}

\section{Computers in Education and Education in Computer Science}

\author{
José Luis Sierra-Rodríguez ${ }^{1}$, Francisco José García-Peñalvo ${ }^{2}$ \\ 'Departamento de Ingeniería del Software e Inteligencia Artificial, Universidad Complutense de Madrid, Madrid, España. Jlsierra@fdi.ucm.es \\ Grupo GRIAL, Departamento de Informática y Automática, Facultad de Ciencias, Universidad de Salamanca, Salamanca, España. \\ fgarcia@usal.es
}

Resumen

Selección de artículos extendidos relacionados con Informática Educativa y Educación en Informática presentados en la decimosexta edición del Simposio Internacional de Informática Educativa (SIIE 2014), celebrado entre el 12 y el 14 de noviembre de 2014 en Logroño, La Rioja, España.

\begin{abstract}
Selection of the extended papers related to Computers in Education and Computer Science Education topics presented at the sixteenth edition of the International Symposium on Computers in Education (SIIE 2014), held between 12 and 14 November 2014 in Logroño, La Ri-oja, Spain.
\end{abstract}

Keywords:

Palabras Clave:

Computers in Education; Education in Computer Science. 


\section{Introducción}

Esta sección especial integra las versiones revisadas y extendidas de cuatro trabajos sobre Informática Educativa y Educación en Informática presentados en SIIE'14 (Sierra Rodríguez, Dodero Beardo, \& Burgos, 2014a, 2014b), la decimosexta edición del Simposio Internacional de Informática Educativa celebrado entre el 12 y el 14 de noviembre de 2014 en Logroño, La Rioja, España.

SIIE es uno de los eventos de referencia en la aplicación de las Tecnologías de la Información y de las Comunicaciones a la Educación en el ámbito Iberoamericano. El Simposio ofrece un foro internacional para la presentación y debate de los últimos avances en investigación sobre las tecnologías para el aprendizaje y su aplicación práctica en los procesos educativos. También pretende poner en contacto a investigadores, desarrolladores, representantes institucionales y profesores para compartir puntos de vista, conocimientos y experiencias.

La edición SIIE'14 tuvo como tema central el acceso masivo y universal a recursos educativos como soporte al aprendizaje a lo largo de la vida. Con ello el simposio pretendió hacerse eco del enorme auge adquirido en los últimos años por enfoques que, como los representados por los cursos masivos en abierto (MOOC) (Fidalgo Blanco, García-Peñalvo, \& Sein-Echaluce Lacleta, 2013; Fidalgo
Blanco, Sein-Echaluce Lacleta, Borrás Gené, \& García-Peñalvo, 2014; Fidalgo Blanco, Sein-Echaluce Lacleta, \& García-Peñalvo, 2013, 2015; García-Peñalvo, Fidalgo Blanco, \& Sein-Echaluce Lacleta, 2014; SCOPEO, 2013; Zapata-Ros, 2013), suponen una democratización real y de ámbito mundial al acceso a materiales educativos de calidad en abierto. El principal objetivo del simposio fue, de esta manera, permitir avanzar en la forma en la que los métodos, técnicas y herramientas de la informática educativa contribuyen a promover el acceso inclusivo, personalizado y a lo largo de la vida a un patrimonio cultural universal.

Al igual que en ediciones pasadas, SIIE'14 integró la Informática Educativa y la Educación en Informática como líneas temáticas vertebrales (Dodero et al., 2014; Dodero et al., 2013; García-Peñalvo, Sarasa Cabezuelo, \& Sierra González, 2014; GarcíaPeñalvo, Sarasa Cabezuelo, \& Sierra Rodríguez, 2014; Velazquez-Iturbide \& Garcia-Penalvo, 2009). Además, el simposio desarrolló los eventos ISELEAR (Ingeniería del Software en eLearning) y SPDECE (Simposio Pluridisciplinar sobre Diseño y Evaluación de Contenidos Digitales Educativos) como líneas adicionales e integradas en el mismo. Por último, junto con las presentaciones de artículos científicos, el Simposio ofreció un conjunto de talleres y sesiones temáticas. 


\section{Contenidos de la sección especial}

En relación con las líneas básicas desarrolladas en SIIE'14 que nos ocupan en esta sección especial:

- La línea temática sobre Informática Educativa articuló los temas relativos a la aplicación de las tecnologías de la información y las comunicaciones en educación en general. Dicha línea desarrolló aspectos tales como el análisis del aprendizaje, la aplicación de teorías pedagógicas al diseño de software educativo, las aplicaciones de la web semántica en educación, los modelos de aprendizaje apoyado en la tecnología (aprendizaje a distancia, online e híbrido, aprendizaje colaborativo, aprendizaje con sistemas móviles/ubicuos, etc.), la autoría de contenidos educativos, los cursos masivos en abierto (MOOC), el conocimiento abierto en contextos educativos, el diseño y estandarización de lenguajes de modelado y metadatos educativos, el desarrollo y evaluación de software educativo, la e-Evaluación, los entornos personalizados para el aprendizaje, la formación de profesores en las TIC, la gestión de las competencias en los contextos educativos, la inteligencia artificial en educación, la interacción persona-computador en contextos educativos, los laboratorios virtuales y laboratorios remotos, las metodologías y experiencias de uso de software educativo, la minería de datos educativos, la multimedia, hipermedia y visualización en educación, los mundos virtuales educativos, la robótica educativa, los sistemas de cursos, herramientas y recursos basados en web, los sistemas y plataformas de gestión educativa, el soporte del aprendizaje no formal e informal ligado al puesto de trabajo, los videojuegos y simulaciones educativas, o la web social y las comunidades de aprendizaje.

- La línea temática sobre Educación en Informática agrupó los temas relativos a dicho aspecto específico de la Informática Educativa, incluyendo métodos y herramientas para la educación en campos específicos de la Informática, y estrategias de enseñanza y aprendizaje del pensamiento computacional más allá de las disciplinas específicas de Informática. Los temas originalmente contemplados por esta línea incluyeron, entre otros, el diseño y evaluación de herramientas para la enseñanzaaprendizaje en materias específicas de informática, las visualizaciones y animaciones para educación en informática, las simulaciones y juegos serios para educación en informática, el diseño curricular en informática, la informática en enseñanzas secundarias y el pensamiento computacional, la 
enseñanza-aprendizaje de materias propias de la informática (programación, bases de datos, sistemas operativos, arquitectura de computadores, lenguajes de programación y construcción de compiladores, etc.), la informática en ciencias experimentales, sociales y humanas, los métodos y casos de estudio en Platform-Based Development (web, móviles, etc.), las estrategias de aprendizaje en informática, o los cursos masivos en abierto en informática.

La edición SIIE'14 recibió un total de 60 contribuciones. Tras un proceso de revisión por pares exhaustivo, en el que cada contribución fue revisada por, al menos, tres miembros del comité de programa, 34 fueron aceptadas como "artículo largo", otras 9 fueron aceptadas como "artículo corto", y 2 fueron aceptadas como propuesta de taller. Los trabajos aceptados ofrecieron, de esta forma, una completa y actualizada perspectiva del estado actual y las tendencias en Informática Educativa en cada una de las dimensiones abordadas en el Simposio. Esta sección especial contiene, por tanto, las versiones extendidas y revisadas de cuatro de los trabajos más notables presentados en el simposio, y que abordan aspectos seleccionados de la Informática Educativa y de la Educación en Informática. Más concretamente:

- En "La docencia como un fractal: de la experiencia al modelo" Patricia Compañ, Rafael Carmona, Rosana Satorre y Faraón
Llorens (2015) parten de la opinión de los estudiantes sobre aspectos que influyen directamente sobre la calidad de la docencia (masificación, carga de trabajo asociado a las materias, los tipos de aulas en las que se desarrollan las clases, etc.), así como de su propia experiencia como docentes en relación con distintas prácticas docentes (presentaciones orales en clase, estrategias de corrección basadas en pares, etc.) para proponer un modelo que mejore la motivación del alumnado. Dicho modelo, que tiene en cuenta aspectos clave como la motivación, la evaluación, la sensación de progreso y el aprendizaje autónomo a fin de propugnar la retroalimentación y fomentar la motivación del estudiante, se basa en elementos de diseño instruccional así como en analogías con los principios de la geometría fractal.

- En "Rubric-based tools to support the monitoring and assessment of Bachelor's Final Projects" Verónica Moreno y Davinia Hernández (2015) abordan el problema crítico del seguimiento y evaluación de los proyectos fin de grado en Ingeniería. Para ello, las autoras proponen un enfoque basado en rúbricas (es decir, en un conjunto de criterios y estándares utilizados para llevar a cabo el seguimiento y la evaluación de los citados proyectos), y lo evalúan mediante una experiencia llevada a cabo en la Escuela Superior Politécnica de la Universidad Pompeu Fabra de Barcelona. 
- En "Reutilización de datos abiertos en el aprendizaje de diseño de bases de datos a través de proyectos" José Norberto Mazón, Elena Lloret, Eva Gómez, Antonio Aguilar, Iván Mingot, Enesto Pérez, y Luisa Quereda (2015) proponen una estrategia a la enseñanza y el aprendizaje de materias relacionadas con bases de datos basada en el empleo de cuerpos de datos abiertos disponibles públicamente. Para ello, los alumnos deben proponer aplicaciones que reutilicen distintos bancos de datos abiertos para un determinado fin. Los estudiantes deben, entonces, diseñar bases de datos que faciliten la gestión de dichos datos para los propósitos abordados por las aplicaciones, y poblar dichas bases de datos a partir de los bancos de datos abiertos seleccionados. Como resultado, se aumenta la motivación de los alumnos, que valoran el uso de datos reales como vehículo para llevar a cabo proyectos relacionados con las citadas materias de bases de datos.

- Por último, en "Uso de GitHub en el diseño de e-actividades para la refactorización del software" Carlos López, Jesús M. Alonso, Raúl Marticorena y Jesús M. Maudes (2015) se centran en el importante aspecto de la enseñanza y el aprendizaje de la refactorización de software. Para ello, proponen un conjunto de e-actividades de refactorización de código Java organizadas de acuerdo a la taxonomía de Bloom, y soportadas por las funcionalidades de versionado del repositorio de código abierto GitHub. Los autores analizan, así mismo, el impacto de su propuesta tanto desde el punto de vista del efecto en el aprendizaje, como desde el punto de vista de la carga de trabajo que supone tanto para el docente como para el estudiante.

Todos estos trabajos han sido sustancialmente revisados y extendidos por sus autores, y han superado un exhaustivo proceso de revisión por pares que ha involucrado varios ciclos de revisión, de acuerdo con los estándares de la revista "Education in the Knowledge Society".

Los editores invitados quieren agradecer a los comités directivos y de programa de SIIE'14, a la Asociación Española para el Desarrollo de la Informática Educativa (ADIE), así como a los autores de los cuatro trabajos que integran el número especial y a los revisores que, desinteresadamente, han intervenido en el proceso de revisión, por la estupenda labor científica realizada. Así mismo, quieren agradecer a todo el equipo de "Education in the Knowledge Society" por el soporte proporcionado y por la oportunidad brindada para organizar este número especial. 


\section{Referencias}

Compañ-Rosique, P., Molina-Carmona, R., Fidalgo Blanco, Á., Sein-Echaluce Lacleta, Satorre-Cuerda, R., \& Llorens-Largo, F. M. L., Borrás Gené, O., \& García-Peñalvo, F. (2015). Teaching as a fractal: From experience J. (2014). Educación en abierto: Integración to model. Education in the Knowledge Society, de un MOOC con una asignatura académica. 16(4), 32-46. http://dx.doi.org/10.14201/ Education in the Knowledge Society (formerly eks20151643246

Revista Teoría de la Educación: Educación y Cultura en la Sociedad de la Información),

Dodero, J. M., García-Peñalvo, F. J., 15(3), 233-255. Retrieved from http:// González, C., Moreno-Ger, P., Redondo, campus.usal.es/ revistas_trabajo/index. M. Á., Sarasa, A., \& Sierra, J. L. (2014). php/revistatesi/article/view/12226/12571

Development of E-Learning Solutions:

Different Approaches, a Common Mission. Fidalgo Blanco, Á., Sein-Echaluce Lacleta, IEEE Revista Iberoamericana de Tecnologías M. L., \& García-Peñalvo, F. J. (2013). del Aprendizaje (IEEE RITA), 9(2), 72-80. MOOC cooperativo. Una integración entre h t t p : / / d x.d o i. org/10.1109/ cMOOC y xMOOC. In Á. Fidalgo Blanco \& RITA.2014.2317532

M. L. Sein-Echaluce Lacleta (Eds.), Actas del II Congreso Internacional sobre Aprendizaje,

Dodero, J. M., García-Peñalvo, F. J., Innovación y Competitividad (CINAIC) (pp. González, C., Moreno-Ger, P., Redondo, M. 481-486). Madrid, Spain: Fundación General Á., Sarasa-Cabezuelo, A., \& Sierra-Rodríguez, de la Universidad Politécnica de Madrid.

J. L. (2013). Desarrollo de Soluciones para E-Learning: Diferentes Enfoques, un Objetivo Común. VAEP-RITA, 1(2), 113-122.

Fidalgo Blanco, Á., Sein-Echaluce Lacleta, M. L., \& García-Peñalvo, F. J. (2015). Methodological Approach and Technological Fidalgo Blanco, Á., García-Peñalvo, F. Framework to break the current limitations J., \& Sein-Echaluce Lacleta, M. L. (2013). of MOOC model. Journal of Universal A methodology proposal for developing Computer Science, 21(5), 712-734. adaptive cMOOC. In F. J. García-Peñalvo (Ed.), Proceedings of the First International García-Peñalvo, F. J., Fidalgo Blanco, Á., Conference on Technological Ecosystems for \& Sein-Echaluce Lacleta, M. L. (2014). Enhancing Multiculturality (TEEM'13) (pp. Tendencias en los MOOCs. Retrieved 553-558). New York, NY, USA: ACM. from http://gredos.usal.es/jspui/ http://dx.doi.org/10.1145/2536536.2536621 handle/10366/125093 
García-Peñalvo, F. J., Sarasa Cabezuelo, A., \& Sierra González, J. L. (2014). Innovating in the Engineering Processes: Engineering as a Means of Innovation. IEEE Revista Iberoamericana de Tecnologías del Aprendizaje (IEEE RITA), 9(4), 131-132. http://dx.doi. org/10.1109/RITA.2014.2363004

García-Peñalvo, F. J., Sarasa Cabezuelo, A., \& Sierra Rodríguez, J. L. (2014). Innovando en los Procesos de Ingeniería. Ingeniería como Medio de Innovación. VAEP-RITA, 2(1), 2628.

López, C., Alonso, J. M., Marticorena, R., \& Maudes, J. M. (2015). Uso de GitHub en el diseño de e-actividades para la refactorización del software. Education in the Knowledge Society, 16(4), 81-96. http:// dx.doi.org/10.14201/eks20151648196

Mazón, J. N., Lloret, E., Gómez, E., Aguilar, A., Mingot, I., Pérez, E., \& Quereda, L. (2015). Reutilización de datos abiertos en el aprendizaje de diseño de bases de datos a través de proyectos. Education in the Knowledge Society, 16(4), 63-80. http:// dx.doi.org/10.14201/eks20151646380

Moreno Oliver, V., \& Hernández-Leo, D. (2015). Rubric-based tools to support the monitoring and assessment of Bachelor's Final Projects. Education in the Knowledge Society, 16(4), 47-62. http://dx.doi.org/10.14201/ eks20151644762

SCOPEO. (2013). SCOPEO INFORME No.2: MOOC: Estado de la situación actual, posibilidades, retos y futuro. Retrieved from Salamanca, Spain: http://scopeo.usal.es/wpcontent/uploads/2013/06/scopeoi002.pdf Sierra Rodríguez, J. L., Dodero Beardo, J. M., \& Burgos, D. (2014a). International Symposium on Computers in Education (SIIE), 2014. USA: IEEE.

Sierra Rodríguez, J. L., Dodero Beardo, J. M., \& Burgos, D. (2014b). XVI Simposio Internacional de Informática Educativa (SIIE'14). Acceso masivo y universal para un aprendizaje a lo largo de la vida (12-14 de Noviembre de 2014, Logroño, La Rioja, España). España: Universidad Internacional de La Rioja (UNIR).

Velazquez-Iturbide, J. A., \& Garcia-Penalvo, F. J. (2009). Computers in Education: Advances in Software Technology. Journal of Universal Computer Science, 15(7), 14231426.

Zapata-Ros, M. (2013). MOOCs, una visión crítica y una alternativa complementaria: La individualización del aprendizaje y de la ayuda pedagógica. Campus Virtuales. Revista Científica Iberoamericana de Tecnología Educativa, 2(1), 20-38. Retrieved from http://www.revistacampusvirtuales.es/ campusvirtuales/2/2.pdf 